\title{
Traitement antiparasitaire des maladies parodontales et péri-implantites : suivi multicentrique ò 12 mois
}

Antiparasitic treatment of periodontitis and
peri-implantitis: 12-months multicentric follow-up

\section{MOTS-CLES :}

- Parodontite, amibe, Entamoeba, biofilm, microscope, PCR, implant dentaire, péri-implantite.

\section{KEYWORDS:}

- Parodontitis, amoebae, Entamoeba, biofilm, microscope, $P C R$, dental implant, peri-implantitis.

AOS 2013;261:22-28 DOI: $10.1051 / \mathrm{aos} / 2013104$ (C) EDP Sciences 2013

\section{Résumé}

Depuis plus d'un siècle, les chercheurs ont identifié le protozoaire Entamoeba gingivalis dans le sillon des dents atteintes par les maladies parodontales. L'utilisation récente de réaction en chaîne par polymérase (PCR) a permis de détecter cette amibe dans $69 \%$ des sites malades et démontré son absence dans la situation de gencive saine. Le but de cet article est de visualiser les caractéristiques d'un tel biofilm et d'évaluer les résultats cliniques de cabinets dentaires concernés par la composante parasitaire dans les thérapies parodontales et implantaires.

\section{Abstract}

For nearly a century, researchers have identified the protozoan parasite Entomoeba gingivalis from diseased gingival pocket. Recent use of PCR detected the parasite in 69\% of diseased sites and demonstrated its absence in healthy gingival sites. The purpose of this cases report was to visualise this characteristic biofilm and evaluate periodontal results of dental clinics concerned with parasitic component of periodontal and peri-implantitis therapy.

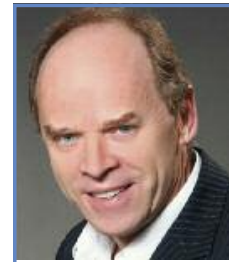

- Mark BONNER, Docteur en médecine dentaire, Institut international de parodontie, 455, Promenade-des-Anglais, Arenas, Le Nice Premier, o6200 Nice.

Véronique AMARD, Docteur en chirurgie dentaire, 94100 St-Maur-des-Fossés. Marion VERDY, Docteur en chirurgie dentaire, 75015 Paris.

Pierre AMIOT, Docteur en chirurgie dentaire, 66000 Perpignan.

Mathieu MARTY, Docteur en chirurgie dentaire, 660oo Perpignan.

Jean-Pierre ROCHET, Docteur en chirurgie dentaire, 93150 Le Blanc-Mesnil.

Serge IHLER, Docteur en chirurgie dentaire, Cabinet Philippe Leclercq, 75008 Paris.

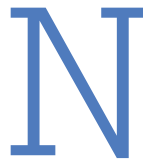

os connaissances sur les organismes parasites et leur relation avec les maladies humaines sont beaucoup moins connues que celles des bactéries. Les auteurs du début du siècle
[1] relatent qu'ils trouvent l'amibe Amoeba dentalis dans l'inflammation gingivale et qu'elle ressemble à Amoeba coli de l'intestin humain ; Barret, en 1914, annonce plus spécifiquement qu'il trouve Entamoeba 
gingivalis dans presque tous les cas de pyorrhée alvéolaire qu'il a examinés. Il avance l'hypothèse que la présence de cette amibe est d'une importance significative dans la maladie parodontale. Les résultats bénéfiques à la suite de l'administration d'émétine hydrochloride les mènent à assigner un rôle pathologique à cet organisme ; l'année suivante, ils prolongent létude et confirment ces observations sur plus de 300 cas. Dans leur travail, ils assument continuellement que les parasites vivent à l'intérieur et aux dépens du tissus gingival et de l'os alvéolaire ; ils rapportent aussi que l'amibe est retrouvée en plus grande quantité à l'extrémité apicale de la poche alvéolaire.

Entamoeba gingivalis présent dans les maladies parodontales est facilement transmis par des gouttelettes d'infection et une partie infime de salive portant cet organisme peut être infectieuse [2]. Il devient alors possible de transmettre ce parasite depuis le partage de couverts et de produits cosmétiques aussi bien que par contact direct. Kofoid [1] cite Hinshaw et Sinonton qui concluent en affirmant que l'amibe n’a jamais été trouvée dans une bouche strictement normale et est invariablement trouvée dans une parodontite typique de ses tous débuts jusqu'à sa fin. L'existence et l'approfondissement de la poche gingivale accompagnent invariablement ce type d'inflammation. De plus, les patients qui prennent bon soins de leur bouche semblent tout aussi susceptibles de présenter l'infection amibienne. Augmentant avec l'âge, l'incidence de l'infection parasitaire progresse rapidement jusquà ce qu'au-delà de 40 ans elle atteigne $75 \%$ de la population. Les études subséquentes se font plutôt descriptives.

Un autre niveau de sensibilité et de spécificité a pu être atteint par l'utilisation de la méthode de réaction en chaîne par polymérase (PCR). En premier, l'identification PCR de E. gingivalis [3] a échoué à démontrer clairement la présence d'amibes chez les patients, avec seulement $6 \%$ déchantillons positifs. La faible sensibilité de la paire d'amorce a été démontrée par Trim et al. [4], qui ont utilisé une méthode de purification d'ADN plus appropriée, atteignant $27 \%$ d'échantillons positifs chez les patients avec la même amorce, alors qu'ils ont obtenu $69 \%$ avec un ensemble de paire d'amorces et un PCR en temps réel qui lui est plus précis. Il apparaît important de noter qu'E. gingivalis n'a été détectée dans aucune bouche saine ni dans aucune dent saine de patients affectés par une maladie parodontale localisée.

Un dentiste canadien, le Dr Trevor Lyons [5-8], suite à l'observation microscopique du biofilm creviculaire de sa patientèle pendant plus de 10 ans, remarque lévidence de l'invasion du sillon parodontal par E. gingivalis. Son mode de diagnostic et de traitement est basé sur l'approche médicale diagnostique et thérapeutique du parasite intestinal E. histolytica. Ce clinicien rapporte lui aussi que les parasites oraux sont trouvés dans tous les cas de parodontites, qu'ils se nourrissent de globules blancs autant que de globules rouges et qu'une amélioration clinique notable est invariablement obtenue par leur élimination. Puisque les amibes, lorsque présentes, sont trouvées de façon constante à la base de la poche infectée, les échantillons sont collectés essentiellement dans ces endroits spécifiques. Les parasites oraux sont trouvés seulement dans les sites malades. Il en conclut que, malgré l'adage qu'E. gingivalis soit un commensal, le poids de l'évidence démontre qu'il s'agit là d'un pathogène agressif. Il apparaît donc, tel que décrit dans son ouvrage [8], que la vaste majorité des patients touchés par la maladie parodontale sont infectés par les parasites oraux et que l'élimination de ces protozoaires est suivie par l'arrêt de la maladie et sa résolution, incluant la régénération osseuse.

Tirant profit des connaissances de Lyons, notre expérience clinique pendant 26 ans à la microscopie parodontale démontre généralement que l'hygiène habituelle n'est pas suffisante pour se débarrasser des protozoaires buccaux dans la majorité des cas de parodontites. Dans une étude de Linke [9] conduite chez 10 patients atteints de parodontite avancée, tous les sujets sont porteurs d'E gingivalis. La recommandation pose à l'évidence de prendre plusieurs sites chez le même patient afin d'éviter les faux négatifs. Sa conclusion est que les données rapportées en général dans la littérature sur l'incidence d' E. gingivalis après un seul échantillon par patient est beaucoup trop basse et doit être interprétée avec précaution. E. gingivalis est présente dans $65,4 \%$ des sujets de 30 à 34 ans [10]. La prévalence du protozoaire est beaucoup plus importante en cas de parodontolyse destructrice que chez les sujets apparemment indemnes. On en conclut donc, en 1990, qu'il existe une corrélation entre l'atteinte des tissus parodontaux et l'infestation aux protozoaires buccaux tout comme en 1929 à la suite de l'importante étude de Kofoid [1]. Comme d'autres amibes, E. gingivalis possède une protéine de surface apparentée à la fibronectine [4]. Celle-ci intervient dans les mécanismes d'adhésion et de phagocytose, ce qui l'impliquerait dans les premières phases de destruction des tissus parodontaux.

Plus déterminant encore, diverses formes d'amibes se retrouvent couramment dans l'eau des robinets et dans la tubulure des unités dentaires [11] et certains de ces protozoaires seraient à caractère possiblement pathogène.

\section{MÉTHODE ET RÉSULTATS}

Les données de cette étude sont tirées de nos dossiers cliniques ainsi que de létude rétrospective de 5 cabinets dentaires concernés par la composante parasitaire des maladies parodontales. Nous avons donc procédé à l'instar des recommandations de Lyons, à l'observation des frottis du biofilm creviculaire à létat frais, tirés du plus profond des sillons parodontaux affectés. L'observation au microscope à contraste de phase sur montage salivaire obligatoire, pour éviter la déformation des protozoaires, permet rapidement de constater que tous les patients présentant l'amibe E. gingivalis sont 
atteints à un certain degré de maladie parodontale (fig. 1). Les patients en parfaite santé parodontale exempts de saignement gingival ne présentent pas de parasites mais essentiellement des bactéries de formes cocoïdes et filamenteuses non mobiles (fig. 2) en accord avec les travaux de Sockransky [12] et de Nisengard et Newman [13]. Les capacités d'envahissement et de locomotion de cette amibe sont importantes. Nous avons observé que l'amibe est souvent motile, émettant un pseudopode à sa partie antérieure et présente la formation d'un uroïde à sa partie distale, connu comme phénomène de "capping" [14] (fig. 3). Chez le pathogène humain Entamoeba histolytica, il semble que l'uroïde puisse jouer un rôle dans lévasion de la réponse immunitaire de l'hôte. L'adhésion, l'inquilinisme (fig. 4) et la phagocytose (fig. 5), un autre facteur important relié à la pathogénicité de l'espèce proche E. histolytica, sont facilement observables. Lamibe laisse sur son passage après phagocytose du noyau des polymorphonucléaires neutrophiles une cellule évidée remplie d’enzymes protéolytiques aussi décrite comme cellule fantôme, brisant ainsi le processus habituel d'apoptose [15]. Lablation de leur contenu, et plus précisément de leur noyau, empêcherait la formation de «NET» (Neutrophile Extracellular Trap), des réseaux de fibres de chromatine (structure nucléaire organisée d'ADN et de protéines) exposée hors de la cellule et sur laquelle sont fixées des enzymes, constituant des pièges lytiques pour les pathogènes [16]. Ce processus de phagocytose du noyau par l'amibe (que nous avons appelé exonucléophagie) pourrait être une cause de l'impossibilité du système immunitaire à résoudre l'infection durant les parodontites ou les péri-implantites, amenant ainsi une altération chronique. Lamibe n'est pas anecdotique dans le sillon parodontal des patients (fig. 6) et peut démontrer une forte division ainsi que la formation de véritables nids amibiens (fig. 7).

Tel que proposé par Lyons, la thérapie utilisée vise plus particulièrement à éliminer l'amibiase parodontale. Nous avons donc procédé à des contrôles mensuels en traitant par thérapie locale à l'aide d'eau hydrogénée et de poudre salée de bicarbonate de soude en guise de dentifrice, puis utilisé une crème de métronidazole $10 \%$ en application topique pour une période de quelques mois, et enfin complété en dernier ressort par une médication antiparasitaire systémique afin d'éliminer définitivement l'amibiase si persistante.

Invariablement, les signes cliniques de guérison des parodontites sur dents naturelles apparaissent et le biofilm infectieux s'estompe pour laisser place à une flore normale exempte de neutrophiles, de bactéries mobiles et de parasites. Une fois cette flore pathogène éliminée, les saignements ayant disparu, la rétraction de la gencive s'effectue rapidement, puis le tartre résiduel plus aisément observable est éliminé. Nous avons obtenu en 2003 [17] chez un groupe de 20 nouveaux patients atteints de parodontite avancée et agressive ayant accompli cette thérapie progressive, l'élimination de $94 \%$ des poches parodontales excédant

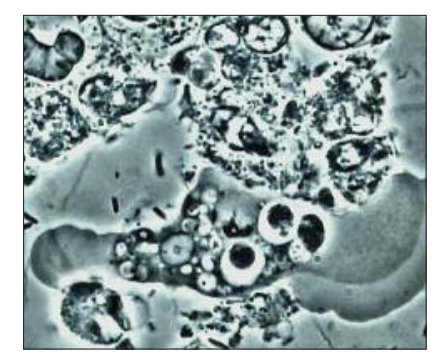

$\triangleleft$ Fig. 1 :

(Grossissement 1000 x) Amibe typique entourée de polynucléaires neutrophiles, bacilles et spirillés. E. gingivalis se distingue par son noyau arrondi vers la gauche, le karyosome central et la chromatine au pourtour du noyau. Un pseudopode bien formé est visible sur la gauche et un pseudopode lamellaire au pourtour du cytoplasme à droite. Trois vacuoles rappellent l'ingestion récente de noyaux de leucocytes.

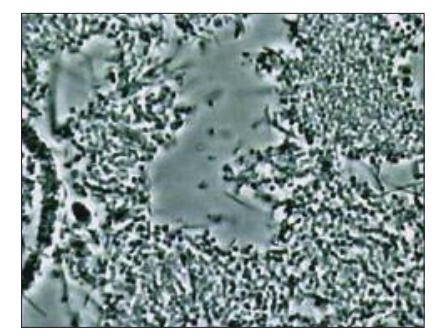

4 Fig. 2 :

Flore normale chez un patient en bonne santé parodontale. Bactéries non motiles de forme coccoïde, accompagnées de filaments de diverses longueurs. Sur la gauche un filament entouré de coccoïdes rappelle Corynebacterium matruchotii. On note l'absence de cellules inflammatoires.

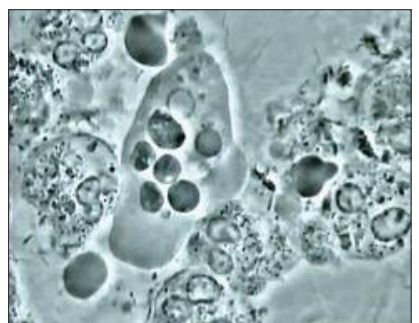

4 Fig. 3 :

Une amibe d'allure plutôt vivace accompagnée de bactéries motiles converge vers une cellule granulocyte. Les vacuoles digestives composées des noyaux phagocytés des PMN sont de bonnes dimensions et bien visibles. La partie distale présente un uroïde conforme avec le phénomène dit de « capping».

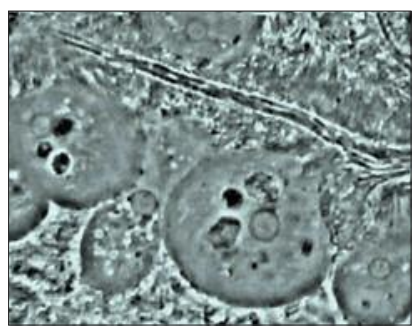

Fig. 4 :

E. gingivalis en contact intime avec des grands filaments bactériens formant palissade, indiquant une forte adhésion dans un cas ici de parodontite lié à la grossesse. La notion d'organisation et d'inquilinisme laisse suspecter un élément avancé d'ergonomie chez ces parasites.

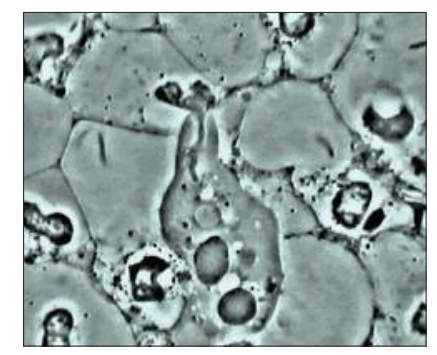

Fig. 5 :

Début de la phagocytose du noyau du leucocyte polymorphonucléaire par une amibe dans un cas de parodontite active. Notez la formation intracytoplasmique plus dense du noyau du granulocyte qui est progressivement ingéré. Au pourtour, on remarque des carcasses fantômes des cellules blanches évidées, rejets dénucléés hors contrôle qui se dissiperont sur les tissus environnants.

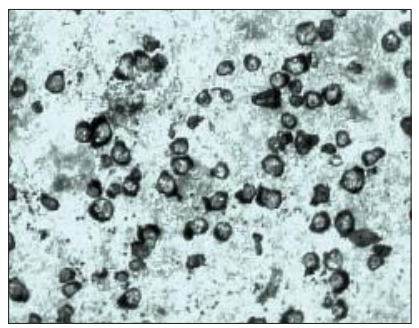

Fig. 6 :

(Grossissement $100 \mathrm{x}$ ) Présence d'une cinquantaine d'amibes sur un champ à fond noir. Les portions plus foncées font foi de l'aspect des pseudopodes alors que la partie plus pâle concentre la portion digestive et vacuolaire de l'endoplasme.

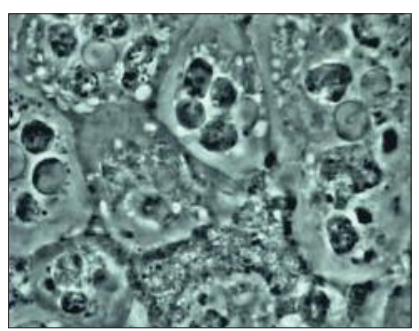

Fig. 7 :

Amas de parasites dans un cas de parodontite à progression rapide suggérant une nidification importante d'E. gingivalis rappelant vivement l'abcès amibien du foie et la destruction des tissus environnants. Six amibes trophozoïtes baignent sur un fond purulent. 


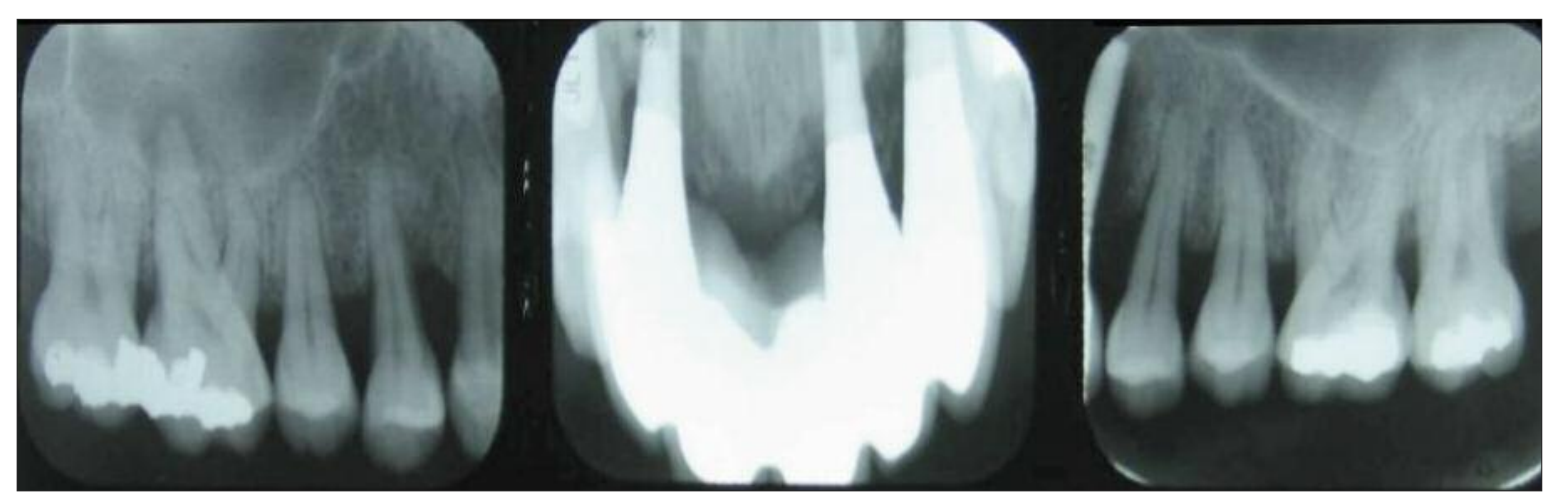

$\triangleleft$ Fig. 8 :

Radiographie d'un cas de péri-implantite visible par la perte osseuse verticale en situation de l'implant $n^{\circ} 23$.

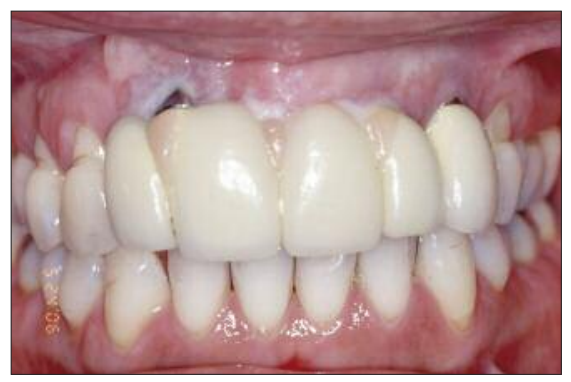

4 Fig. 9 :

Même patient en cours de thérapie parodontale antiparasitaire traité à lorigine par la pose d'implants dentaires antérieurs dans une situation de parodontite non résolue depuis 15 ans.

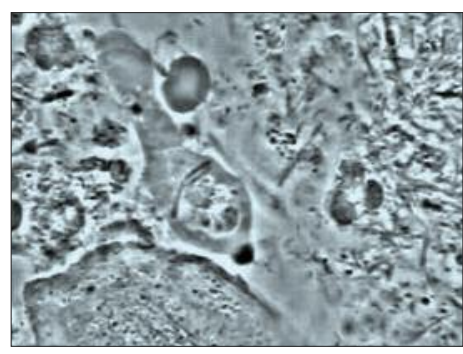

$\triangleleft$ Fig. 10 :

Biofilm dans ce cas de péri-implantite avec présence de polymorhonucléaires neutrophiles et amibe unipolaire remontant vers le haut de l'image présentant un uroïde à son extrémité distale.

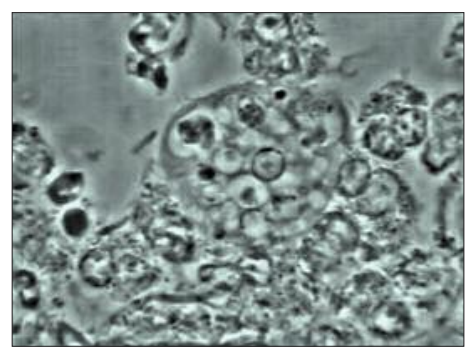

$\triangleleft$ Fig. 11 :

Même cas de péri-implantite avec une amibe au centre avec son noyau caractéristique, ses vacuoles digestives constituées de noyaux phagocytés et entourée de PMN.

$3 \mathrm{~mm}$, calculé selon un format plus descriptif pour le clinicien proposé par Harrell et Nunn [18], après la première année. La flore initiale typiquement pathogène composée de parasites, neutrophiles, spirochètes et bactéries motiles est remplacée par une flore exempte de globules blancs, constituée de bactéries coccoïdes et filaments immobiles comme dans tous les cas de santé parodontale. La guérison est stable dans le temps et les facteurs de réinfection sont contrôlés suite à la thérapie : entourage, conjoints, famille proche, animaux de compagnie, voyages dans les zones à risques, vecteurs de transmission indirects, à l'instar de toute maladie parasitaire de même nature.

Nous avons en 2011 complété une étude rétrospective dans cinq cabinets français utilisant ce protocole de traitement antiparasitaire Lyons-Bonner-Dunoyé. Dans ces relevés cliniques, pour 632 patients traités pour parodontite chronique ou agressive, sur une base d'un sondage en 6 points par dent, où la normale est considérée à $3 \mathrm{~mm}$ ou moins, nous avons constaté après traitement parodontal antiparasitaire une cicatrisation clinique sulculaire moyenne des poches infectées de 95,7\% pour l'ensemble des patients à 12 mois.

Dans le même ordre d'idée, 32 patients atteints de péri-implantite furent évalués pour la microscopie du biofilm à l'aide d'un échantillon à l'état frais. À titre d'exemple, l'image radiologique typique (fig. 8) et clinique (fig. 9) de l'implant en situation $\mathrm{n}^{\circ} 23$ en cours de thérapie faite par le patient montre une perte osseuse verticale approchant l'apex de l'implant. À l'origine, une pression sur la gencive laisse évacuer un suintement jaunâtre habituel de l'infection parodontale. À la microscopie, l'image de l'examen à l'état frais de départ (fig. 10 et 11) rappelle exactement le biofilm de la parodontite chronique ou agressive avec la présence d'amibes et de globules blancs polynucléaires neutrophiles en activité ou ayant fait apoptose.

\section{DISCUSSION}

Les chercheurs utilisant le microscope à contraste de phase ont identifié le protozoaire parasite E. gingivalis à partir des poches parodontales infectées depuis près de 100 ans. Les études récentes ont développé une approche de biologie moléculaire pour déterminer la présence d'E. gingivalis dans les poches parodontales malades et vérifier les sites gingivaux en santé. Pour cela, une PCR conventionnelle [3] avait été utilisée avec des pointes de papier insérées dans les poches parodontales saines ou en maladie parodontale. Plus récemment, Trim et al. [4] ont amélioré les sondes PCR et obtenu des données de présence des protozoaires se rapprochant des données microscopiques cliniques. Ainsi cette dernière technique se montre dorénavant plus adéquate pour évaluer la présence d'E. gingivalis. Aucun protozoaire n'a été détecté dans aucun des sites sains de la gencive ni avec l'une ou l'autre des différentes PCR.

Cette nouvelle méthodologie permet d'ajouter un nouveau marqueur eucaryote du statut de la poche gingivale. De plus, aucune amibe n'a été détectée dans une poche saine d'un patient diagnostiqué comme ayant une maladie parodontale localisée. On rapporte la possibilité qu'E. gingivalis puisse élaborer des enzymes protéolytiques qui contribueraient à la 

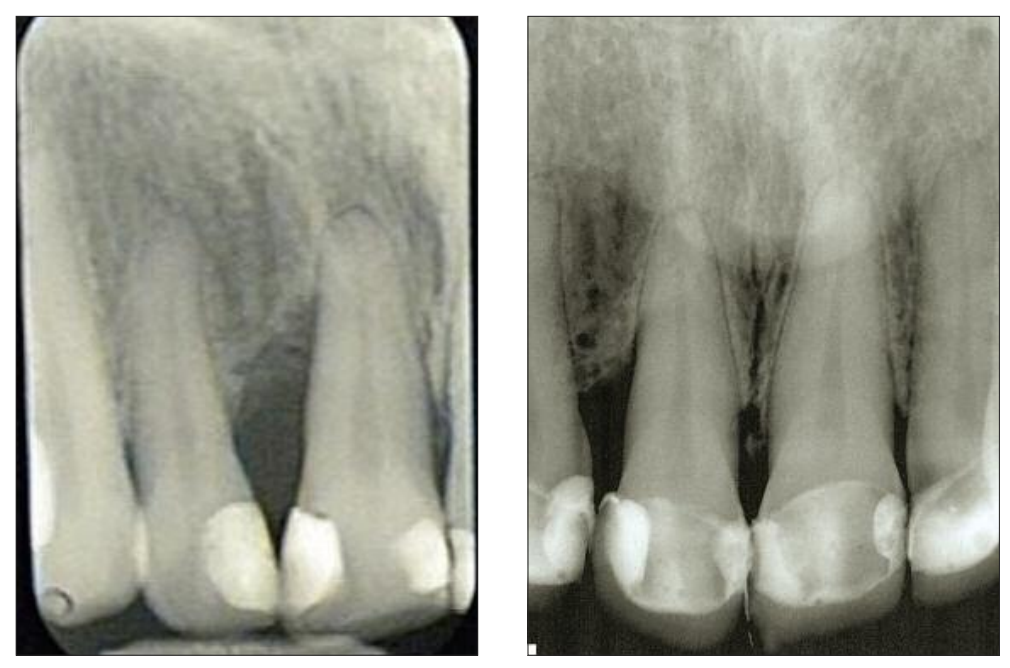

$\triangle$ Fig. 12 :

Radiographie pré-traitement parodontal antiparasitaire avec mobilité I de la dent $n^{\circ} 11$. Notez le défaut angulaire au distal de cette dent.

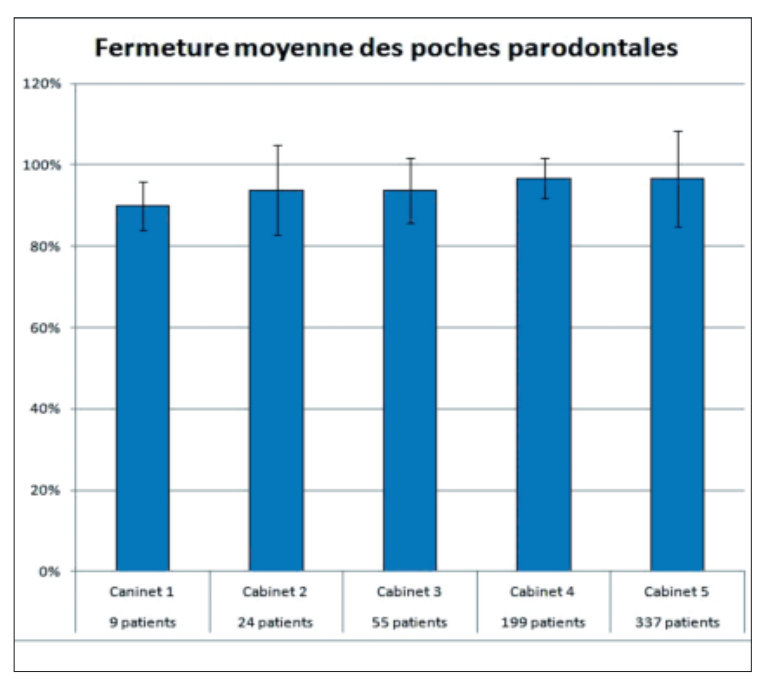

$\triangle$ Tableau I :

Dans 5 cabinets de type différent : pratiques généralistes (cabinets $1,2,3$ ), pratiques vouées à la parodontie (cabinets 4,5 ) les résultats de la fermeture des poches parodontales suite à la mise en place du protocole antiparasitaire sont sensiblement les mêmes avec un haut degré de guérison parodontale quel que soit l'achalandage.

pathogenèse de la parodontite. Les résultats de cette dernière investigation amènent une base rationnelle pour aider à comprendre le rôle étiologique potentiel de ces parasites.

Bien que la maladie parodontale occupe toujours l'attention des chercheurs médicaux et dentaires et bien que les facteurs locaux, les facteurs systémiques, les habitudes comme celles du fumeur, aient été impliqués dans le processus de la maladie parodontale, le succès global dans les thérapies parodontales non chirurgicales a souvent été décevant avec une perte annuelle de $0,15 \mathrm{~mm}$ [18]. Aujourd'hui encore, la modalité de traitement prédominant est d'ordre mécanique et encourage l'ablation d'une partie des tissus oraux. Sans le concours de relevés microbiens, le résultat initial apparaît favorable ; cependant, la déception est souvent inévitable.
Plus paradoxal face aux techniques d'implantation, nous avons observé la présence de ces parasites accompagnés de polymorphonucléaires neutrophiles à la microscopie dans la situation clinique de perte osseuse dans les cas de défaut à long terme d'intégration implantaire. Dans un cabinet particulier concerné par les péri-implantites, l'évaluation microscopique des patients a permis de visualiser la présence de l'amibe chez 31 patients sur un total de 32. La thérapie parodontale du cas de péri-implantite présenté a permis de diminuer la profondeur des poches parodontales sur les dents naturelles sauf sur l'implant touché qui a dû être enlevé suite à des réinfections successives parasitaires et pour cause de mobilité à 6 ans.

Le dénominateur le plus commun dans toutes les parodontites chroniques, agressives et péri-implantites demeure, pour ceux qui veulent bien l'observer, la présence des parasites buccaux. Notre expérience clinique montre que leur élimination scrupuleuse et complète amène sur les dents naturelles une guérison rapide et indolore, fiable dans le temps et permet de compléter la lithotritie résiduelle avec aisance (Tableau I). Le réattachement du ligament parodontal se fait à la façon de toute cicatrisation semblable du corps humain et les défauts osseux angulaires peuvent se combler au fil des années (fig. 12 et 13) même sur des dents initialement vouées à l'extraction, la greffe osseuse et la pose d'implant. Leur élimination dans les cas de périimplantites semble au contraire beaucoup plus difficile ou sinon impossible en regard de la présence d'une inflammation persistante [19] constituée de PMN fournissant ainsi un apport constant en nourriture aux trophozoïtes par la voie de l'exonucléophagie. Tout cela semble confirmer l'habilité potentielle d'E. gingivalis à envahir et détruire les tissus gingivaux inflammés fragiles, premièrement touchés par les bactéries de la gingivite. Rappelons que, chez nos confrères parasitologues, la destruction des tissus du foie est causé par les restes dénucléés des polynucléaires après phagocytose. Selon Orozco [20] et Akuffo [15], la dégénération et la lyse cellulaire amènent la libération de protéases, comme les phospholipides, la collagénase et une cystéine protéase, capable de dégrader la laminine, la fibronectine et le collagène. Notons qu'E. gingivalis peut contenir fréquemment entre 3 et 5 noyaux phagocytés mais ce phénomène peut facilement dépasser plus de 10 (fig. 14). Une seule amibe peut aussi phagocyter jusquà 4 noyaux de neutrophiles à la fois par 4 pseudopodes différents (fig. 15).

À lopposé, un patient dont le biofilm est constitué d'une flore commensale parfaite (bactéries coccoïdes, filaments immobiles et absence de polynucléaires) pourra sans nul doute, dans cette situation de terrain idéal, augmenter ses chances de succès de préparation à la greffe osseuse et à la pose d'implants dentaires dans un contexte où il peut difficilement s'infecter par la voie de contamination directe intra-buccale (fig. 16 à 19).

D'un point de vue parasitaire, le dernier critère essentiel requis par la guérison parodontale demeure 


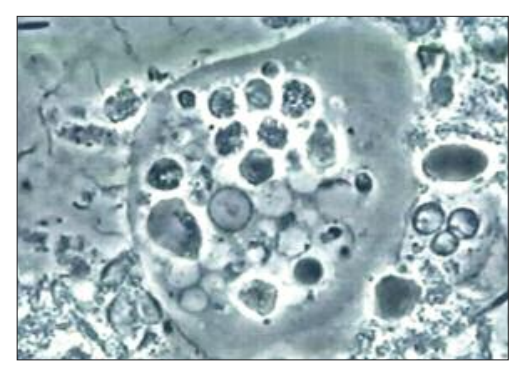

4 Fig. 14 :

Amibe présente dans un cas de parodontite agressive. Notez suite à la phagocytose des noyaux des PMN plus d'une dizaine de masses en cours de digestion dans l'endoplasme amibien. Au centre du trophozoïte le noyau distinctif avec son karyosome central et les grains de chromatine au pourtour.

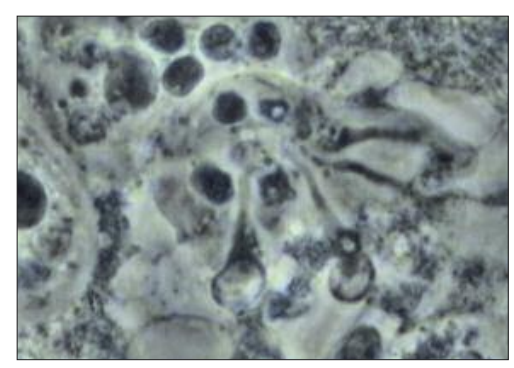

$\triangleleft$ Fig. 15 :

Large amibe en pleine activité de phagocytose simultanée, dévorant 4 noyaux de cellules neutrophiles à la fois et laissant au pourtour des cellules blanches dénucléées logiquement hors contrôle. être une première étape pour en comprendre sa biologie et ses interactions avec l'hôte humain [23]. De nombreux aspects cliniques et biomoléculaires similaires à E. histolytica, responsables de l'amibiase intestinale [24, 25, doivent mériter l'attention des chercheurs face à des caractères typiquement pathogènes. De futures études sont encouragées afin de déterminer le génome de ce parasite et ainsi mieux établir ses paramètres biomoléculaires à caractère virulent en comparaison avec les espèces histolytica, dispar et la souche Rahman [23].

E. gingivalis agit dans diverses formes de parodontites incluant les péri-implantites comme un pathogène envahissant à la lueur de son activité hématophage et de la dégradation de l'immunité cellulaire de l'hôte la prévention de la réinfection en éliminant les vecteurs de transport incluant entre autres la gestion microbienne parodontale pré-implantologie, l'assurance de bonnes habitudes orales et la surveillance de l'entourage familial et environnemental tel que stipulé dans le domaine des infections parasitaires.

\section{CONCLUSION}

Les observations actuelles permettent de confirmer que l'amibe E. gingivalis joue un rôle très particulier dans la pathogenèse des parodontites et des périimplantites. Il est raisonnable d'estimer qu'E. gingivalis puisse être l'agent qui permette le passage d'une pathologie bénigne, la gingivite, en une maladie destructrice, la parodontite ou la péri-implantite. De plus, les facteurs typiques de pathogénicité que sont la forte mobilité et les capacités de phagocytose amènent à penser que le trophozoïte E. gingivalis partage des traits de virulence de son proche cousin E. histolytica, tels que la modulation de la réponse de l'hôte et la lyse des tissus environnants. Lamibe E. gingivalis semble manipuler cette réponse en inactivant certains facteurs (tel les neutrophiles dénucléés) qui autrement protégerait l'os maxillaire de la destruction. Sa présence dans le biofilm est une haute constante dans les maladies parodontales et implantaires. E. gingivalis a aussi été diagnostiqué comme responsable d'abcès pulmonaires [21] et retrouvé récemment dans un cas d’ostéomyélite mandibulaire [22]. Son élimination concomitante avec les bactéries anaérobiques assure par voie de ciblage ou de causalité directe une guérison efficace de la parodontite chronique et agressive. Les études comparatives d'autres espèces qui colonisent lêtre humain pourraient aider à comprendre les questions de biologie fondamentale concernant la virulence, les interactions hôte-parasites et les préoccupations de santé publique face à la maladie parodontale et au rejet implantaire. Le séquençage génomique de cette espèce est encouragé et pourrait

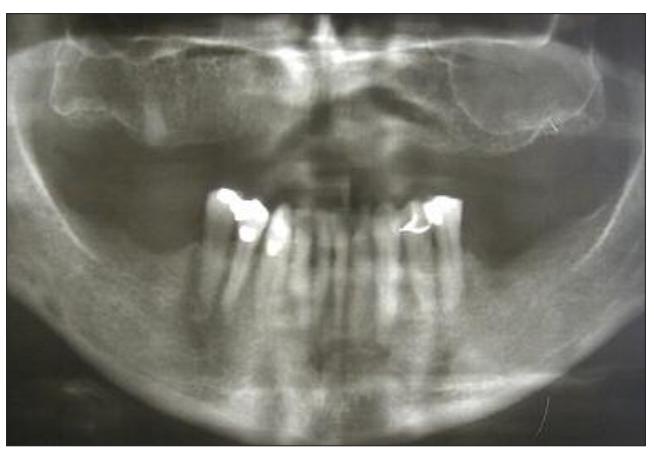

$\triangleleft$ Fig. 16:

Radiographie pré-

opératoire de remplacement des sextants postérieurs par greffe autogène d'apposition et prothèses fixes sur implants dans une situation de biofilm commensal.

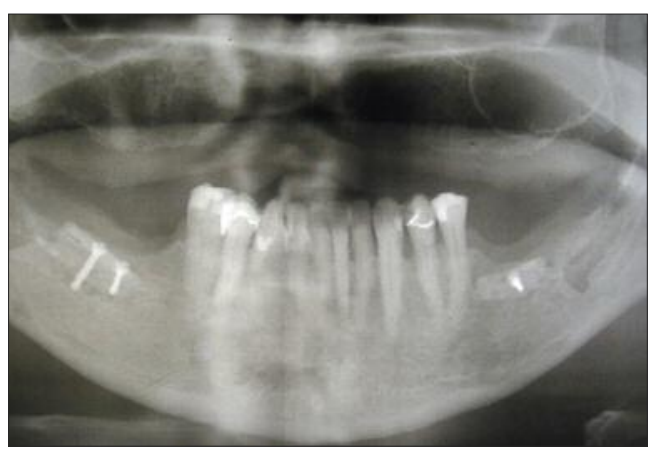

4 Fig. 17 :

Pose de greffons bilatéraux postérieurs à partir de la base des branches montantes.

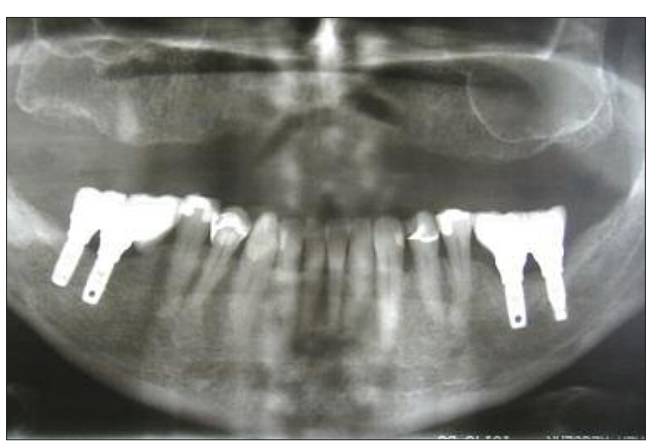

4 Fig. 18 :

Implants et prothèses fixes en place depuis 10 ans.

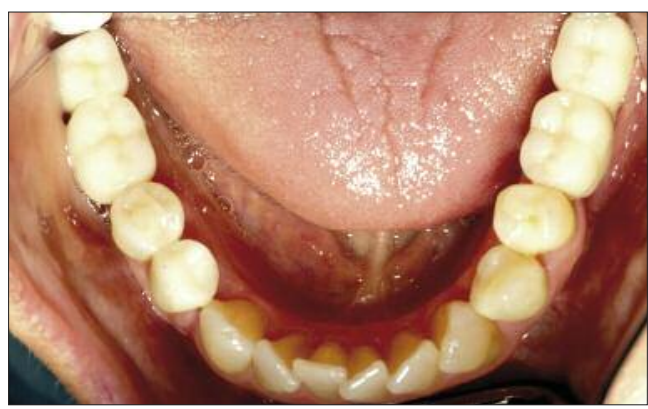

$\varangle$ Fig. 19 :

Situation clinique sous contrôle microscopique biannuel de la bonne maintenance d'une flore microbienne commensale. 
qu'il engendre. Sa détection dans le sillon parodontal devrait susciter une thérapie équivalente antiparasitaire, garantissant ainsi une meilleure chance de succès et un retour à la flore commensale, garante de la santé parodontale ou d'une pose d'implant dentaire dans un environnement pour le moins favorable.

\section{REMERCIEMENTS}

Nous tenons à remercier Julien Santi-Rocca, PhD en biochimie et biologie moléculaire, spécialisé en parasitologie de l'université Pierre et Marie Curie, Paris VI, pour sa contribution et ses conseils pendant cette étude.

Nous remercions également Mme Solange Dunoyé, communicatrice et directrice de l'Institut international de parodontie pour sa contribution à la mise à jour du protocole Bonner-Lyons-Dunoyé.

\section{Bibliographie}

[1] Kofoid CA, Hinshaw HC, Johnstone HG. Animal parasites of the mouth and their relation to dental disease.

J Am Dent Assoc 1929;1436-455.

[2] Koch DA. Relation of moisture and temperature to the viability of Entamoeba gingivalis (Gros) in vitro. Univ Calif Publ Zool 1927;(31):17-29.

[3] Kikuta N, Yamamoto A, Goto N. Detection and identification of Entamoeba gingivalis by specific amplification of rRNA gene. Can J Microbiol 1996;42(12):1248-51.

[4] Trim RD, Skinner MA, Farone MB, Dubois JD, Newsome AL. Use of PCR to detect Entamoeba gingivalis in diseased gingival pockets and demonstrate its absence in healthy gingival sites. Parasitol Res 2011;109(3):857-64

[5] Lyons T, Sholten T, Palmer JC. Oral amoebiasis: a new approach for the general practitioner in the diagnosis and treatment of periodontal disease. Oral Health 1980;70(10): 39-41,108,110.

[6] Lyons T, Sholten T, Palmer JC, Stanfield E. Oral amoebiasis: alternatives in oral disease. Ont Dent 1982;59(2):16-20.

[7] Lyons T, Sholten T, Palmer JC, Stanfield E. Oral amebiasis: the role of Entamoeba gingivalis in periodontal disease. Quintessence Int Dent Dig 1983;14(12):1245-8.

[8] Lyons T. Introduction to protozoa and fungi in periodontal infections. Trevor Lyons publications, Ontario, Canada 1989. ISBN 0-
9693950-0-0 (disponible sur demande : http ://www.parodontite.com)

[9] Linke HA, Gannon JT, Obin JN. Clinical survey of Entamoeba gingivalis by multiple sampling in patients with advanced periodontal disease. Int J Parasitol 1989;19(7):803-8.

[10] Feki A, Molet A. Importance des protozoaires Trichomonas tenax et Entamoeba gingivalis dans la cavité buccale humaine. Rev Odonto Stomatol 1990;19(1):37-45

[11] Barbeau J, Buhler T. Biofilms augment the number of free-living amoebae in dental unit waterlines. Res Microbiol 2001;152(8):753-60.

[12] Sockransky SS, Haffajee AD. Evidence of bacterial etiology: a historical perspective. Periodontol 2000. 1994;5:7-25.

[13] Nisengard RJ, Newman MG. Oral microbiology and immunology. Philadelphia: W.B. Saunders Company, 1994:297.

[14] Arhets P. Gounon P. Sansonetti P. Guillén N. Myosin II is involved in capping and uroid formation in the human pathogen Entamoeba histolytica. Infect Immun 1995;63(11):4358-67

[15] Akuffo H, Linder E, Ljungström I, Wahlgren M. Parasites of the colder climates. New-York: Taylor \& Francis, 2003.

[16] Papayannopoulos V, Zychlinsky A. NETs: a new strategy for using old weapons. Trends Immunol 2009;30(11):513-21 Epub 2009 Aug 21.

[17] Bonner M. Entamoeba gingivalis un pathogène dans les parodontites. Inf Dent
2003;24:1660-6

[18] Harrel SK, Nunn ME. Longitudinal comparison of the periodontal status of patients with moderate to severe periodontal disease receiving no treatment, non-surgical treatment, and surgical treatment utilizing individual sites for analysis. J Periodontol 2001;72(11): 1509-19.

[19] Klinge B, Hultin M, Berglundh T. Peri-implantitis. Dent Clin North Am 2005;49(3):661-76, vii-viii.

[20] Orozco E. Rodriguez AM, Camargo-Baez M. Riveron AM. Biologia molecular de Entamoeba histolytica. In: Genetica y Biomedicina Molecular. Noriega-Limusa SA de CV. Mexico DF. 2000:267-86.

[21] Jian B, Kolansky AS, Baloach ZW, Gupta PK. Entamoeba gingivalis pulmonary abscess diagnosed by fine needle aspiration . Cytojournal 2008;30;5:12.

[22] Bhaijee F, Bell D. Entamoeba gingivalis in Acute Osteomyelitis of the Mandible. Case Report Med 2011;2011:357301.

[23] Olivos-García A, Saavedra E, Ramos-Martínez E, Nequiz M, Pérez-Tamayo R. Molecular nature of virulence in Entamoeba histolytica. Infect Genet Evol 2009;9(6):1033-7.

[24] Espinosa-Cantellano M, Martínez-Palomo A. Pathogenesis of intestinal amebiasis: from molecules to disease. Clin Microbiol Rev 2000;13(2):318-31.

[25] Martinez-Palomo A. The pathognesis of amebiasis. Parasitology Today 1987;3(4):111-8 